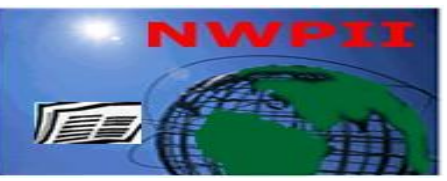

American Journal of Biomedical Sciences

ISSN: 1937-9080

nwpii.com/ajbms

\title{
Android Smart Phones Application in Tele-monitoring Electrocardiogram (ECG)
}

\author{
Duong Trong Luong*, Nguyen Thai Ha, Nguyen Duc Thuan
}

Department of Electronic Technology and Biomedical Engineering, Hanoi University of Science and Technology,

Vietnam

*Corresponding Author

Duong Trong Luong

Department of Electronic Technology and Biomedical Engineering

Hanoi University of Science and Technology

Vietnam

Email: luong.duongtrong@hust.edu.vn

\begin{tabular}{l|l|l} 
Received:01 November 2018; & Revised:27 November 2018; & Accepted: 08 March 2019
\end{tabular}

\begin{abstract}
This article presents the research and application of android smart phones to support tele-monitoring cardiovascular disease. The smart phones are more flexible and easier to carry than laptops. According to a report from Newzoo, by November 2017, 75\% of smartphones in the worldwide installed with Android operating system. In this paper, the authors designed a system that combined hardware with applications android smart phones software for measuring Electrocardiogram (ECG) signal. The results shown that the convenience and the effect of the application of android smart phones in monitoring cardiovascular disease in particular and healthcare in general, as android smartphone are widely used throughout the world.
\end{abstract}

Keywords:Tele-Health care, Android smartphones, ECG signal, Tele-monitoring, Personal Digital Assistant

\section{Introduction}

Every year in the world tens of millions of people suffer from cardiovascular diseases ${ }^{[1], ~[2]}$. Among them, many patients are in critical condition due to myocardial infarction and stroke for the diagnosis and treatment of late cardiovascular disease. In Vietnam, every 3 adults had one people at risk of cardiovascular disease. According to the Vietnam Association of Cardiologists, 2017, Vietnam will have to $1 / 5$ the population of cardiovascular disease and hypertension. Cardiovascular diseases in the world in general and in Vietnam in particular have been tending to increase rapidly, with more complications with different types of complications and the average age of cardiovascular patients also are diminishing, shifted towards those of working age. This status is too download for the national hospital at Vietnam with the number of the quantity of the day as day, in which the development and the physical maker is provided for the doctor and nurse can follow for response. In addition, cardiopulmonary bypass surgery (ECG) is a basic procedure for identifying patients with non-cardiovascular conditions such as ischemia, arrhythmias, etc. Thus, the provision of 
telemedicine services, such as follow-up services, health care is the trend of the world. Since then, the patient will receive the medical services remotely via phone, laptop or other devices that support monitoring and health care at home without having to go to the hospital except in cases of urgency. This monitoring also helps physician updates on patients more often and give better advice.

Recently, in the world and in Vietnam there are many studies on remote sensing and cardiovascular diagnostic systems using wireless technology such as Zigbee, WiFi, GPRS / 3G and bluetooth for active patient, within the confines of a department, clinic or hospital, remote patient (at home or outside or in the workplace) to patients in an emergency ${ }^{[3-10]}$. This article covers the study of android applications on smart phones to support remote cardiovascular monitoring. With smart phone, it is more flexible, easier to carry than laptops. According to a report from Newzoo, by November 2017, 75\% of smartphones worldwide installed the Android operating system. In addition, the International Data Corporation (IDC) report estimates accounted for $85.1 \%$ android devices total smartphones shipped in 2017. Content articles including parts: the first general introduction to service remote health care and a number of studies have been published relating to the cardiovascular system of remote monitoring using wireless technologies; the implementation of research; the results of the study and finally the conclusions.

\section{Implement}

Block diagram of the tele-monitoring and supports cardiovascular disease diagnosis system using wireless technology in combination with the Internet shown in Figure 1.

The proposed system is applied to the patient in a ward, clinic and treatment in the hospital; distant, at home, at work, or outside, and especially for patients in need of emergency care. These patients are fitted with a compact module for collecting, processing and transmitting cardiac signals using radio technology to the heart for monitoring and processing.

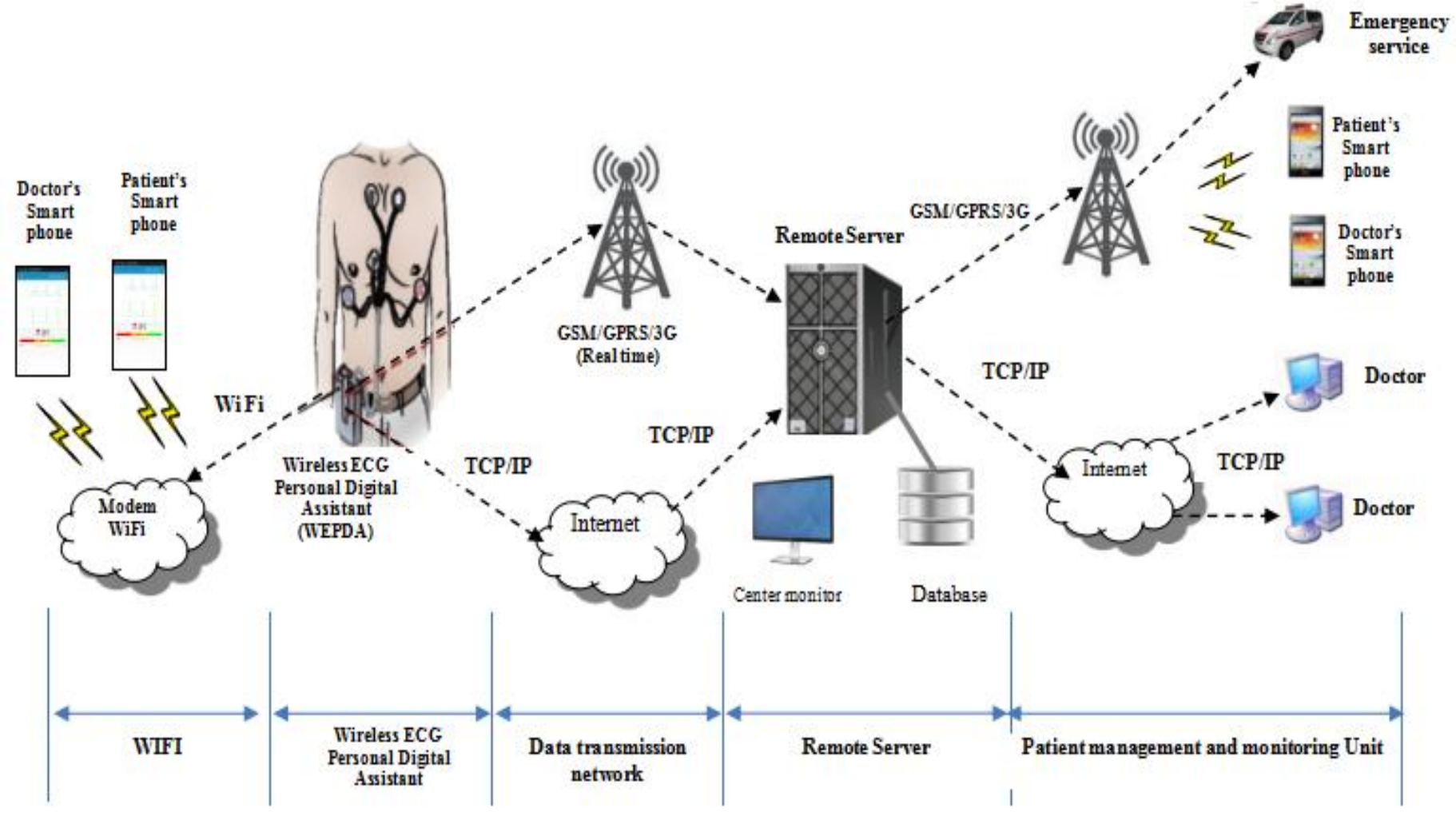

Figure 1: Block diagram of the Tele-monitoring and supports cardiovascular disease diagnosis system using wireless technology in combination with the Internet 
This module, also known as the Personal Digital Assistant (PDA), plays an important role in the local and remote cardiovascular diagnostics and support systems. PDA technology has been developed in recent years and is continuing to be studied to improve the performance and quality of data processing, reduce power generation to save battery life, custom of PDA. Depending on the design of the PDA where it can communicate with other devices in the radio standards such as Zigbee standards, WiFi, GPRS / 3G and bluetooth. PDA performs ECG signal measurement and processing; The PDA then transmits the ECG signal to the processing center or emergency center or to the doctor terminal. Terminal of physician usually smart phones or laptops. The diagram of the ECG display software on the smartphone is shown in Figure 2.

To display the ECG signal and Heart rate information, the authors programming on android app.

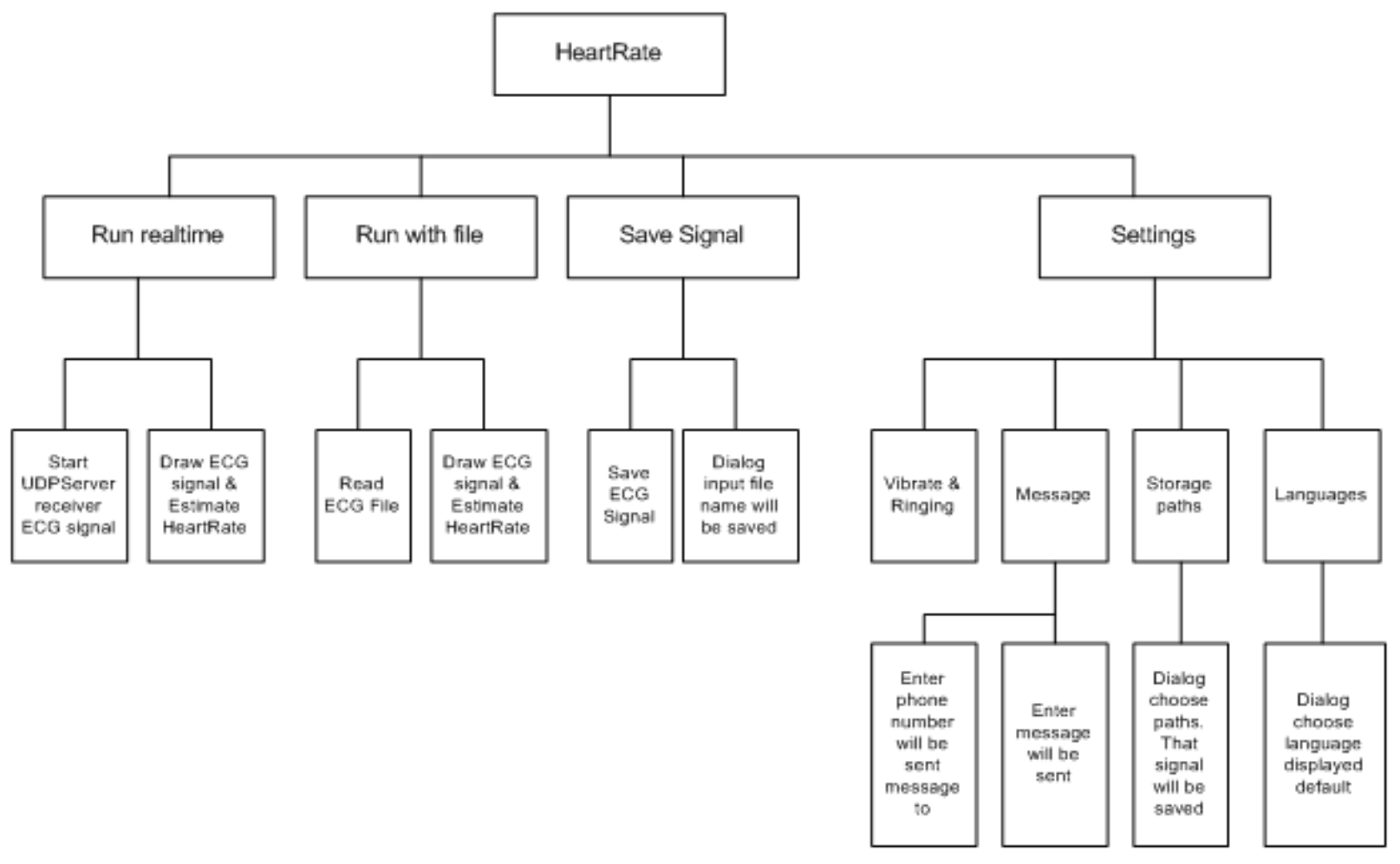

Figure 2: Function diagram of ECG display software on smartphone

In this paper, to test the research methodology, the authors use hardware module to create simulation ECG signal ${ }^{[11]}$.
The ECG signal that the authors use for testing is created by hardware module ${ }^{[11]}$, from Lead I with frequency is $1.2 \mathrm{~Hz}$ (Equivalence Heart Rate is 72 ) and it's form as Figure 3.

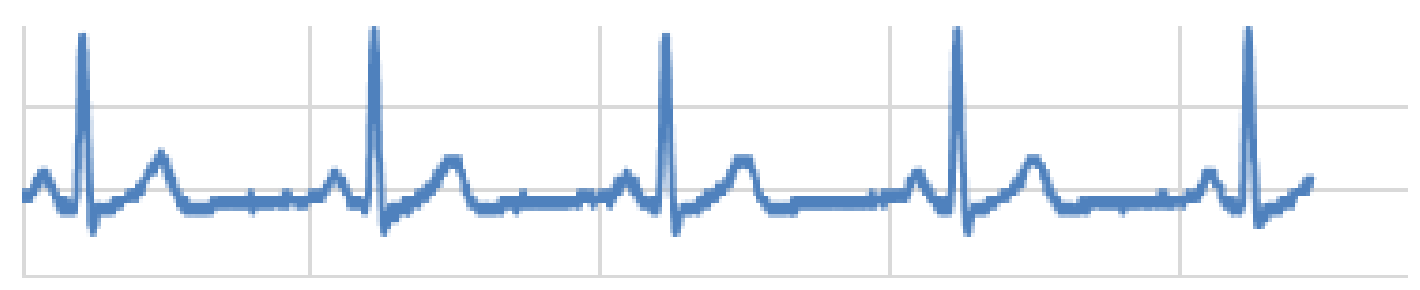

Figure 3: The ECG signal form used for this research's testing 
This hardware module connects to a module with WiFi standard to signal transmission.
Flowchart algorithm for calculating heart rate is shown in Figure 4.

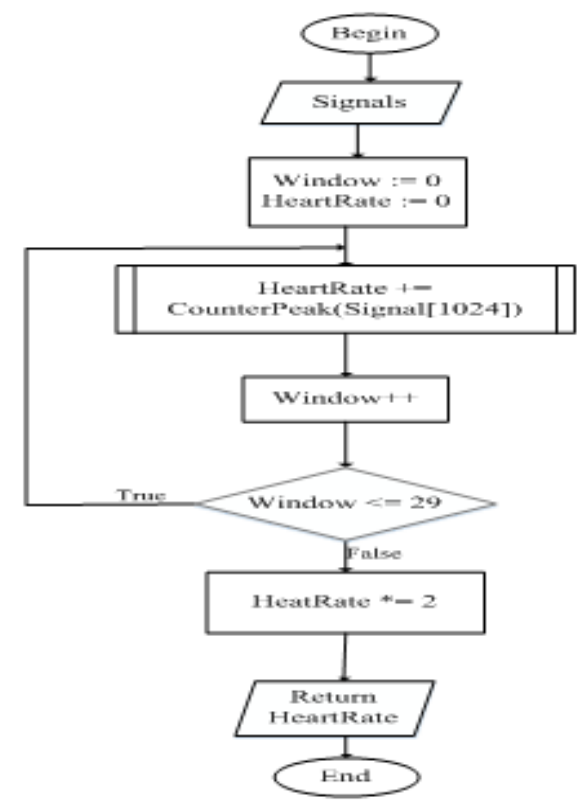

Figure 4: Flowchart algorithm for calculating heart rate

Input: a 30s signal frame is divided into windows with 1024 samples for one window for processing

Output: required heart rate

Algorithm for calculating heart rate is done by following steps

Step1: Creating count variable

Count variable for the total number of signal peaks (HeartRate $)=0$

Count variable for the number of order signal windows is included in the algorithm, Window $=0$

Step 2: Calculate the number of peaks in the window segment with 1024 samples taken through the peaks count function was built above: NumberPeak $=$ counterPeak $($ Signal[1024] $)$. Then sum HeartRate $+=$ Number Peak.

Step 3: Increment window: Window $+=1$

Step 4: Compare Window with 29 (Because of the signal segment calculating in 30s and $\mathrm{x} 2$ )

If Window $<=29$ then back Step 2

Else Window $>29$ continue Step 5

Step 5: Because of the signal segment calculating in 30 s, the heart rate is calculated by the number of signal peaks in $30 \mathrm{~s} x 2$

HeartRate $*=2$
Step 6: Giving heart rate values and end the program

The algorithm for drawing the ECG signal after receiving is shown in Figure 5.

\section{Results}

When users or patients need to measure their own ECG signal and heart rate, they click on a file named "Heart Rate" on the screen of the smartphone. After clicking on this file, the results display screen is shown in Figure 6. This results display screen includes the ECG signal display window, the heart rate calculating window, the display heart rate and status bar alarm the heart rate.

The optional function interface of the software program is shown in Figure 7. The user or patient can choose one of the functions of software programs such as the "Run Realtime" (this is the default function), the "Run with file" (this is the measurement function in offline mode), the function "Save" and the "Settings".

When the user clicks on the "Run Realtime" function menu (this is the default measurement function), the ECG signal software program interface in real time is shown in Figure 8. In this 
program interface includes the ECG form and Heart rate. For the ECG form, it is quite the same as the ECG signal form used for this research's testing (the original ECG signal). The received heart rate by algorithm is $74 \mathrm{bpm}$; so heart rate's error is $1.02 \%$ approximately.

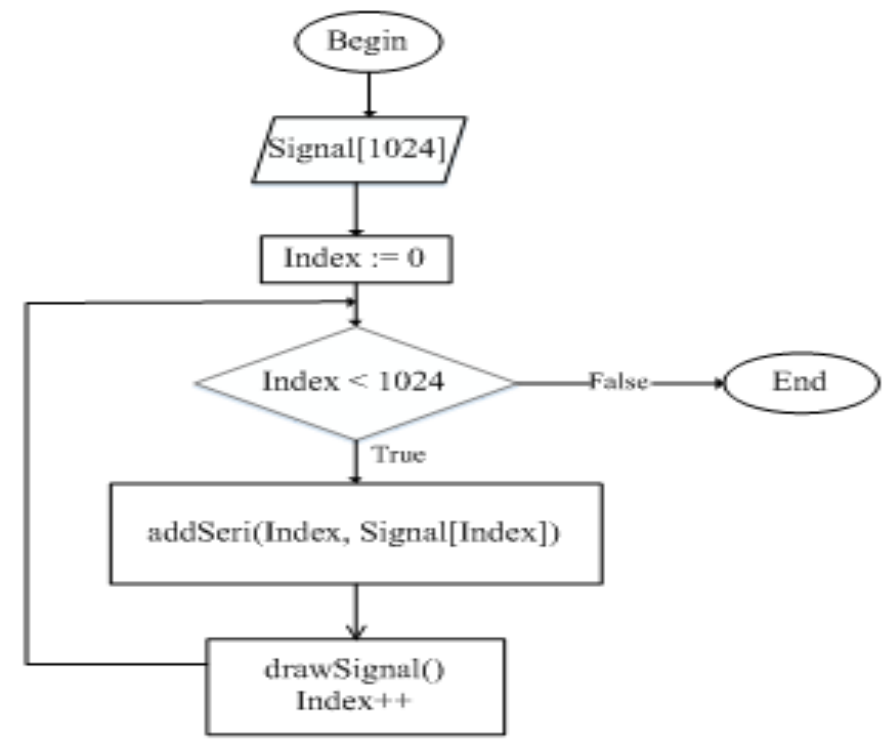

Figure 5: Flowchart algorithm for drawing ECG signal

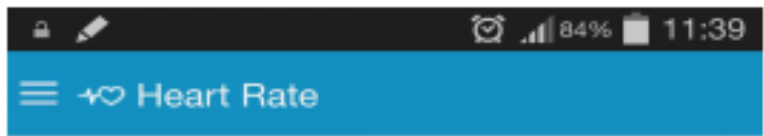

No chart data available.

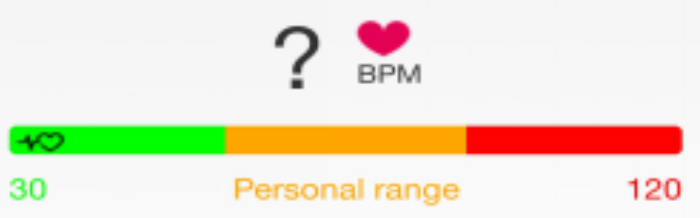

Figure 6:The main interface screen of the application android smartphone display ECG signal measurement results 


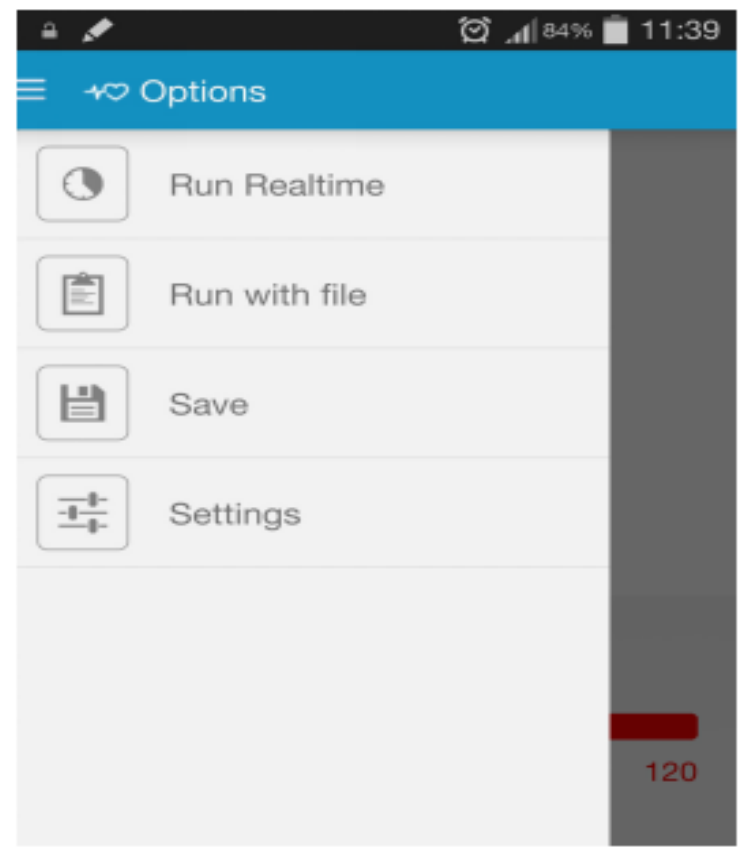

Figure 7: The optional function interface of the android smartphone application software program displays the ECG signal measurement results

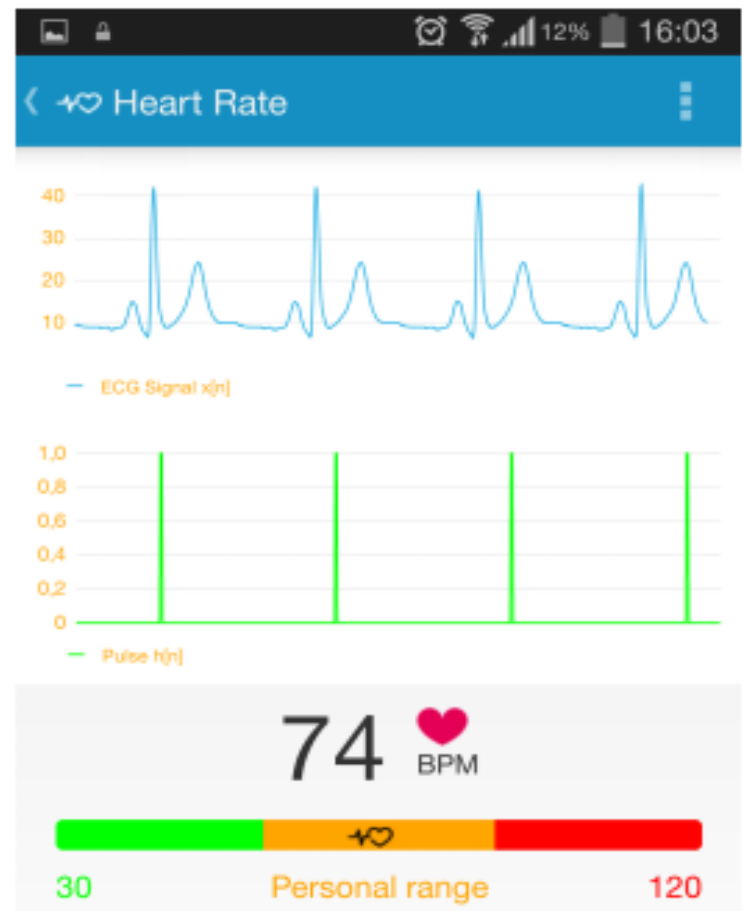

Figure 8: The program interface of the android smartphone application displays the results of the ECG signal in real time

\section{Conclusion}

This article presents an Android smartphone application research to measure and display ECG signals. This is an essential research application in the field of human health care from a distance, at home. The authors have designed a hardware system in combination with the android smartphone 
application software for ECG signal measuring. The proposed system enables Doctor to view patient's ECG on his or her own portable devices. In this system, PDA plays a very important role in receiving ECG signals from the human body and wireless transmitting with some standards such as WiFi, Bluetooth. This device can cost quite expensive (several thousand dollars), in the process of testing, the authors use the ECG signal generator module and connect to the module with WiFi standard. Testing results show the convenience and effective of the application of android smartphones for monitoring ECG signal in particular and general health care when android smartphones are used widely in the world.

\section{References}

1 http://www.cardionet.vn

2 Khalid Abu Al-Saud, Massudi Mahmuddin and Amr Mohamed, Wireless Body Area Sensor Networks Signal Processing and Communication Framework: Survey on Sensing, Communication Technologies, Delivery and Feedback. Journal of Computer Science 2012, ISSN 1549-3636, 8 (1): pp.121132

3 Bo Yu, Liuqing Yang, Chia-Chin Chong, ECG Monitoring over Bluetooth: Data Compression and Transmission. IEEE Wireless Communication and Networking Conference 2010, ISSN : 1525-3511, pp. 1-5

4 CaiKen, Liang Xiaoyin,Development of WiFi based Telecardiology Monitoring system. IEEE 2010, 978-1-4244-5874-5/10
5 Cohen A , Zigel Y . Compression of multichannel ECG through multichannel longterm prediction. IEEE Eng Med Biol Mag 1998; 17(1): 109-115 [PMID: 9460626]

6 Rasid MF , Woodward B . Bluetooth telemedicine processor for multichannel biomedical signal transmission via mobile cellular networks. IEEE Trans Inf Technol Biomed 2005; 9(1): 35-43 [PMID: 15787005]

7 Sana Ullah, Pervez Khan, N. Ullah Shahnaz SaLeem, Henry Higgins, Kyung Sup Kwak (2009), A Review of Wireless Body Area Networks for Medical Applications. Journal of Communications, Network and System Sciences, pp. 797-803

8 Wei Lin,Real time Monitoring of Electrocardiogram through IEEE 802.15.4 Network. IEEE 2011, 978-1-4577-1591-4/11, pp. 1-6.

9 Xin ge, Dakun Lai, Xiaomei Wu, A real time continuous ECG transmitting Method through GPRS with low power consumption, Department of Electronic Engineering Fudan University Shanghai, China. IEEE 2008 9781-4244-1748, pp. 556-559.

10 Hoang Chu Duc, Luong Duong trong, D.Quang and Thuan Nguyen Duc,Mobile device based HRV analysis system. The 5th International Conference on Ubiquitous and Future Networks (ICUFN) .IEEE Xplore 2013, ISSN 2165-8528, pp. 446-449

11 Duong Trong Luong, Nguyen Duc Thuan, Nguyen Thai Ha,New Design of 12-Lead ECG Simulation Signal System with Simulated Noise Added. International Journal of Soft Computing and Engineering (IJSCE) 2015, ISSN: 2231 -2307, Vol. 4, Issue 6, pp. 126-130. 